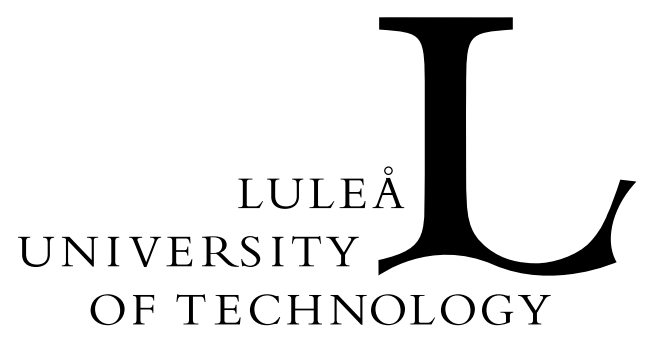

Division of Signal Processing

Visiting address: Universitetsområdet, Porsön, Luleå Postal address: SE-971 87, Luleå, Sweden

Telephone: +46920910 00. Fax: +4692072043

URL: http://www.sm.luth.se/csee/sp/

\title{
OFDM Channel Estimation by Singular Value Decomposition
}

\section{Ove Edfors, Magnus Sandell, Jan-Jaap van de Beek, Sarah Kate Wilson and Per Ola Börjesson}

In IEEE Transactions on Communications, vol.

46 no.7, pp. 931-939, July 1998.

(C) 1998 IEEE. Personal use of this material is permitted.

However, permission to reprint/republish this material for advertising or promotional purposes or for creating new collective works for resale or redistribution to servers or lists, or to reuse any copyrighted component of this work in other works must be obtained from the IEEE. 


\title{
OFDM Channel Estimation by Singular Value Decomposition
}

\author{
Ove Edfors, Associate Member, IEEE, Magnus Sandell, Associate Member, IEEE, \\ Jan-Jaap van de Beek, Student Member, IEEE, Sarah Kate Wilson, Member, IEEE, \\ and Per Ola Börjesson, Member, IEEE
}

\begin{abstract}
In this paper we present and analyze low-rank channel estimators for orthogonal frequency-division multiplexing (OFDM) systems using the frequency correlation of the channel. Low-rank approximations based on the discrete Fourier transform (DFT) have been proposed, but these suffer from poor performance when the channel is not sample spaced. We apply the theory of optimal rank-reduction to linear minimum mean-squared error (LMMSE) estimators and show that these estimators, when using a fixed design, are robust to changes in channel correlation and signal-to-noise ratio (SNR). The performance is presented in terms of uncoded symbol-error rate (SER) for a system using 16-quadrature amplitude modulation (QAM).
\end{abstract}

Index Terms-Channel estimation, OFDM.

\section{INTRODUCTION}

W IRELESS digital communication systems using multiamplitude modulation schemes, such as quadrature amplitude modulation (QAM), generally require estimation and tracking of the fading channel. In general, this means a more complex receiver than for differential modulation schemes, such as differential phase-shift keying (DPSK), where the receivers operate without a channel estimate [1].

In orthogonal frequency-division multiplexing (OFDM) systems, DPSK is appropriate for relatively low data rates, such as in the European digital-audio broadcast (DAB) system [2]. However, for more spectrally efficient OFDM systems, coherent modulation is more appropriate.

The structure of OFDM signaling allows a channel estimator to use both time and frequency correlation. Such a twodimensional estimator structure is generally too complex for a practical implementation. To reduce the complexity, separating the use of time and frequency correlation has been proposed [3]. This combined scheme uses two separate finite-impulse response (FIR) Wiener filters, one in the frequency direction and the other in the time direction.

Paper approved by $O$. Andrisano, the Editor for Fading Channels of the IEEE Communications Society. Manuscript received August 15, 1996; revised July 15,1997 and February 15, 1998. This work was presented in part at the 1996 Vehicular Technology Conference (VTC'96), Atlanta, GA, April 28-May 1, 1996.

O. Edfors is with the Department of Applied Electronics, Lund University, SE-22100 Lund, Sweden.

M. Sandell is with Bell Laboratories, Lucent Technologies, Swindon SN5 6PP, U.K.

J.-J. van de Beek, S. K. Wilson, and P. O. Börjesson are with the Division of Signal Processing, Luleå University of Technology, SE-971 87 Luleå, Sweden.

Publisher Item Identifier S 0090-6778(98)05166-6.
In this paper we present and analyze a class of blockoriented channel estimators for OFDM, where only the frequency correlation of the channel is used in the estimation. Whatever their level of performance, they may be improved with the addition of a second filter using the time correlation [3], [4].

Though the linear minimum mean-squared error (LMMSE) estimator using only frequency correlation has lower complexity than one using both time and frequency correlation, it still requires a large number of operations. We introduce a lowcomplexity approximation to the frequency-based LMMSE estimator that uses the theory of optimal rank reduction [5]. Other types of low-rank approximations, based on the discretetime Fourier transform (DFT), have been proposed for OFDM systems before [6]-[8]. The work presented in this paper was inspired by the observations in [8], where it is shown that DFTbased low-rank channel estimators have limited performance for nonsample-spaced channels and high signal-to-noise ratios (SNR's).

After presenting the OFDM system model and our scenario in Section II, we introduce the estimators and derive their complexities in Section III. We analyze the symbol-error rate (SER) performance in Section IV, where we also discuss design considerations. The proposed low-rank estimator is compared to other estimators in Section $\mathrm{V}$ and a summary and concluding remarks appear in Section VI.

\section{SYSTEM DESCRIPTION}

\section{A. System Model}

Fig. 1 displays the OFDM baseband model used in this paper. We assume that the use of a cyclic prefix (CP) [9] both preserves the orthogonality of the tones and eliminates intersymbol interference (ISI) between consecutive OFDM symbols. Further, the channel is assumed to be slowly fading, so it is considered to be constant during one OFDM symbol. The number of tones in the system is $N$ and the length of the $\mathrm{CP}$ is $L$ samples.

Under these assumptions we can describe the system as a set of parallel Gaussian channels, shown in Fig. 2, with correlated attenuations $h_{k}$. The attenuations on each tone are given by

$$
h_{k}=G\left(\frac{k}{N T_{s}}\right), \quad k=0 \cdots N-1
$$

where $G(\cdot)$ is the frequency response of the channel $g(\tau)$ during the OFDM symbol and $T_{s}$ is the sampling period of 


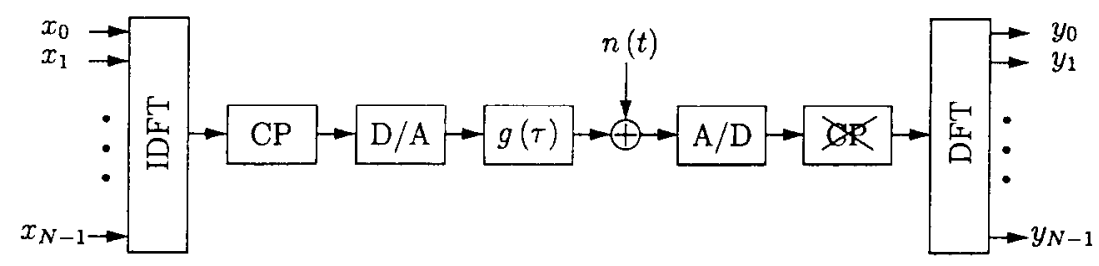

Fig. 1. Baseband model of an OFDM system. CP denotes the cyclic prefix.

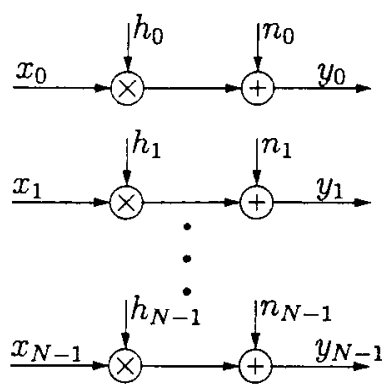

Fig. 2. The OFDM system, described as a set of parallel Gaussian channels with correlated attenuations.

the system. In matrix notation we describe the OFDM system as

$$
y=X h+n
$$

where $\boldsymbol{y}$ is the received vector, $\boldsymbol{X}$ is a diagonal matrix containing the transmitted signaling points, $h$ is a channel attenuation vector, and $n$ is a vector of independent identically distributed (i.i.d.) complex zero-mean Gaussian noise with variance $\sigma_{n}^{2}$. The noise $n$ is assumed to be uncorrelated with the channel $h$.

\section{B. Channel Model}

We consider a fading multipath channel model [1], consisting of $M$ impulses

$$
g(\tau)=\sum_{k=0}^{M-1} \alpha_{k} \delta\left(\tau-\tau_{k} T_{s}\right)
$$

where $\alpha_{k}$ are zero-mean complex Gaussian random variables with a power-delay profile $\theta\left(\tau_{k}\right)$. In this paper we use $M=$ 5 impulses, an exponentially decaying power-delay profile $\theta\left(\tau_{k}\right)=C e^{-\tau_{k} / \tau_{\mathrm{rms}}}$ and delays $\tau_{k}$ that are uniformly and independently distributed over the length of the CP. For correlation properties of this channel model, see Appendix A.

\section{Scenario}

Our scenario consists of a wireless 16-QAM OFDM system, designed for an outdoor environment, that is capable of carrying digital video. The system operates with a $500-\mathrm{kHz}$ bandwidth and is divided into 64 tones with a total symbol period of $136 \mu \mathrm{s}$, of which $8 \mu \mathrm{s}$ constitute the CP. One OFDM symbol thus consists of 68 samples $(N+L=68)$, four of which constitute the $\mathrm{CP}(L=4)$. The uncoded data rate of the system is $1.9 \mathrm{Mb} / \mathrm{s}$. We assume that the root-mean square (rms) width is $\tau_{\mathrm{rms}}=1$ sample $(2 \mu \mathrm{s})$ for the power-delay profile.

\section{LINEAR ESTIMATION ACROSS TONES}

In the following section we present a reduced-complexity LMMSE estimate of the channel attenuations $h$ from the received vector $y$ and the transmitted data $X$. We assume that the received OFDM symbol contains data known to the estimator-either training data or receiver decisions [10]. The proposed estimator can also be used in pilot-symbol assisted modulation (PSAM) [3], where known symbols (pilots) are multiplexed into the transmitted data stream and channel estimation is performed by interpolation; see Section V-B. However, to analyze the properties of the estimator, it is more enlightening to assume that the receiver knows the transmitted data $X$.

The complexity reduction of the LMMSE estimator consists of two separate steps. In the first step we modify the LMMSE by averaging over the transmitted data, obtaining a simplitied estimator. In the second step we reduce the number of multiplications required by applying the theory of optimal rank reduction [5].

\section{A. LMMSE Estimation}

The LMMSE estimate of the channel attenuations $h$ in (1), given the received data $y$ and the transmitted symbols $X$, is [8]

$$
\hat{\boldsymbol{h}}_{\mathrm{lmmse}}=\boldsymbol{R}_{h h}\left(\boldsymbol{R}_{h h}+\sigma_{n}^{2}\left(\boldsymbol{X} \boldsymbol{X}^{H}\right)^{-1}\right)^{-1} \hat{\boldsymbol{h}}_{l s}
$$

where

$$
\hat{\boldsymbol{h}}_{l s}=\boldsymbol{X}^{-1} \boldsymbol{y}=\left[\begin{array}{llll}
\frac{y_{0}}{x_{0}} & \frac{y_{1}}{x_{1}} & \cdots & \frac{y_{N-1}}{x_{N-1}}
\end{array}\right]^{T}
$$

is the least-squares (LS) estimate of $h, \sigma_{n}^{2}$ is the variance of the additive channel noise, and $R_{h h}=E\left\{h h^{H}\right\}$ is the channel autocorrelation matrix. The superscript $(\cdot)^{H}$ denotes Hermitian transpose. In the following we assume, without loss of generality, that the variances of the channel attenuations in $\boldsymbol{h}$ are normalized to unity, i.e., $E\left\{\left|h_{k}\right|^{2}\right\}=1$.

The LMMSE estimator (3) is of considerable complexity since a matrix inversion is needed every time the data in $X$ changes. We reduce the complexity of this estimator by averaging over the transmitted data [1], i.e., we replace the term $\left(X \boldsymbol{X}^{I I}\right)^{-1}$ in (3) with its expectation $E\left\{\left(\boldsymbol{X} \boldsymbol{X}^{I I}\right)^{-1}\right\}$. Simulations indicate that the performance degradation is negligible. Assuming the same signal constellation on all tones and equal probability on all constellation points, we have $E\left\{\left(\boldsymbol{X} \boldsymbol{X}^{H}\right)^{-1}\right\}=E\left\{\left|1 / x_{k}\right|^{2}\right\} \boldsymbol{I}$, where $\boldsymbol{I}$ is the identity matrix. Defining the average SNR as $E\left\{\left|x_{k}\right|^{2}\right\} / \sigma_{n}^{2}$, we obtain the simplified estimator

$$
\hat{h}=R_{h h}\left(R_{h h}+\frac{\beta}{\mathrm{SNR}} I\right)^{-1} \hat{h}_{l s}
$$


where

$$
\beta=E\left\{\left|x_{k}\right|^{2}\right\} E\left\{\left|1 / x_{k}\right|^{2}\right\}
$$

is a constant depending on the signal constellation. In the case of 16-QAM transmission, $\beta=17 / 9$. Because $X$ is no longer a factor in the matrix calculation, the inversion of $R_{h h}+(\beta / \mathrm{SNR}) \boldsymbol{I}$ does not need to be calculated each time the transmitted data in $X$ changes. Furthermore, if $R_{h h}$ and SNR are known beforehand or are set to fixed nominal values, the matrix $\boldsymbol{R}_{l h}\left(\boldsymbol{R}_{h h}+(\beta / \mathrm{SNR}) \boldsymbol{I}\right)^{-1}$ needs to be calculated only once. Under these conditions the estimation requires $N$ multiplications per tone. To further reduce the complexity of the estimator, we proceed with the low-rank approximations below.

\section{B. Optimal Low-Rank Approximations}

Optimal rank reduction is achieved by using the singular value decomposition (SVD) [5]. The SVD of the channel autocovariance matrix is

$$
R_{h h}=U \Lambda U^{H}
$$

where $U$ is a unitary matrix containing the singular vectors and $\boldsymbol{\Lambda}$ is a diagonal matrix containing the singular values $\lambda_{1} \geq \lambda_{2} \geq \cdots \geq \lambda_{N}$ on its diagonal. ${ }^{1}$ In Appendix $\mathrm{B}$ it is shown that the optimal rank- $p$ estimator is

$$
\hat{h}_{p}=U \Delta_{p} U^{H} \hat{h}_{l s}
$$

where $\Delta_{p}$ is a diagonal matrix with entries

$$
\delta_{k}=\left\{\begin{array}{l}
\frac{\lambda_{k}}{\lambda_{k}+\frac{\beta}{\mathrm{SNR}}}, \quad k=1,2, \cdots, p \\
0, \quad k=p+1, \cdots, N .
\end{array}\right.
$$

Interpreting the matrix $U^{H}$ as a transform, ${ }^{2}$ the singular value $\lambda_{k}$ of $\boldsymbol{R}_{h h}$ is the channel power (variance) contained in the $k$ th transform coefficient after transforming the LS estimate $\hat{h}_{l s}$. Since $U$ is unitary, this transformation can be viewed as rotating the vector $\hat{h}_{l s}$ so that all of its components are uncorrelated [5]. The dimension of the space of essentially time- and band-limited signals leads us to the rank needed in the low-rank estimator. In [11] it is shown that this dimension is about $2 B T+1$, where $B$ is the one-sided bandwidth and $T$ is the time interval of the signal. Accordingly, the magnitude of the singular values of $\boldsymbol{R}_{h h}$ should become small after about $L+1$ values, where $L$ is the length of the $\mathrm{CP}(2 B=$ $1 / T_{s}, T=L T_{s}$ and $\left.2 B T+1=L+1\right)$. This is illustrated in Fig. 3, where the singular values of the channel are shown. The system and channel parameters are chosen according to Section II-C. A block diagram of the rank-p estimator in (7) is shown in Fig. 4, where the LS estimate is calculated from $y$ by multiplying by $X^{-1}$. The low-rank estimator can be interpreted as first projecting the LS estimates onto a subspace

\footnotetext{
'Since we are dealing with Hermitian matrices, the $\lambda_{k}$ 's are also eigenvalues. However, we use the terminology of the SVD since it is more general and can be used in optimal rank reduction of nonsquare matrices.

${ }^{2}$ The transform in this special case of low-rank approximation is the Karhunen-Loeve (a.k.a. Hotelling) transform of $h$.
}

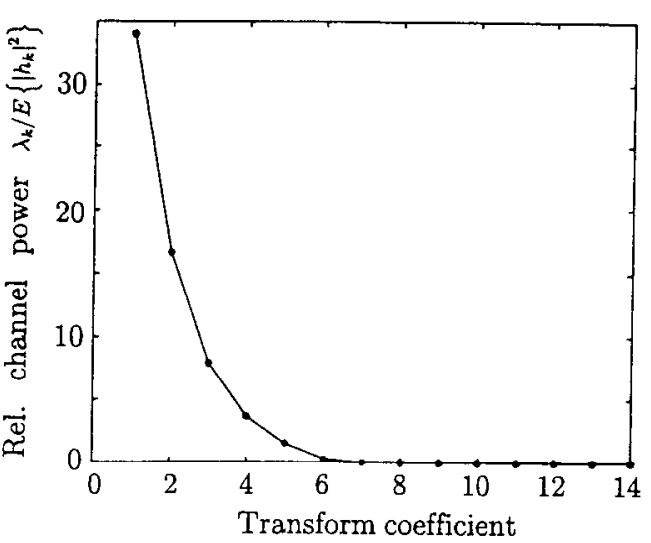

Fig. 3. Relative channel power $\lambda_{k} / E\left\{\left|h_{k}\right|^{2}\right\}$ of the transform coefficients. The system uses 64 tones and the channel parameters are $L=4$ and $T_{\mathrm{r} m \mathrm{~s}}=1$; see Sections II-B and II-C.

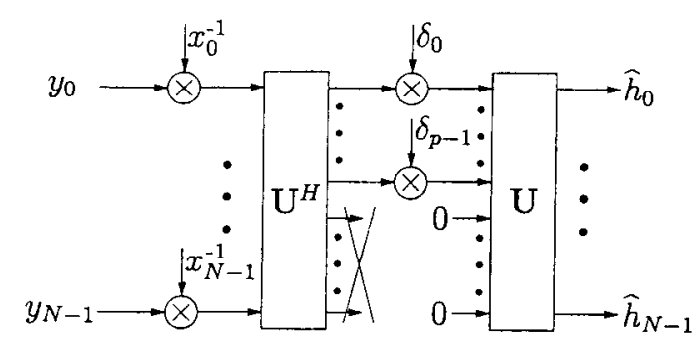

Fig. 4. Block diagram of the rank- $p$ channel estimator.

and then performing the estimation. If the subspace has a small dimension and can describe the channel well, the complexity of the estimator will be low while showing a good performance.

\section{Estimator Complexity}

The low-rank estimators will have an irreducible error floor due to the part of the channel that does not belong to the subspace. To eliminate this error floor up to a given SNR, we need to make sure that our estimator rank is sufficiently large. This prompts an analysis of the computational complexity of the rank- $p$ estimator. In Appendix $C$ the low-rank estimator is shown to require $2 p$ multiplications per tone. In comparison with the estimator (5) we have managed to reduce the number of multiplications from $N$ to $2 p$ per tone. The smaller $p$ is, the lower the computational complexity, but the larger the approximation error becomes. Following the analysis in Section III-B, we can expect a good approximation when $p$ is in the range of the number of samples in the $\mathrm{CP}$, which is usually much smaller than the number of tones $N$.

\section{Partitioning into Subsystems}

A legitimate question at this point is: what happens for a system with many tones and many samples in the CP? The number of calculations per tone can be considerable if a rank- $p$ estimator is used directly on all tones in the system. One solution to this problem is to partition the tones into reasonably sized blocks and, at a certain performance loss, perform the estimation independently in these blocks. By dividing the channel attenuations into $K$ equally sized blocks, the bandwidth in each block is reduced by a factor $K$. 
Referring again to the dimension of the space of essentially time- and band-limited signals [11], the expected number of essential base vectors is reduced from $L+1$ to $L / K+1$. Hence, the complexity of the estimator decreases accordingly.

To illustrate the idea, let us assume a system with $N=1024$ tones and an $L=64$-sample $\mathrm{CP}$. The uniform channel correlation between the attenuations $h_{m}$ and $h_{n}$ in this system is (see Appendix A)

$$
r_{m, n}=\left\{\begin{array}{l}
1, \quad \text { if } m=n \\
\frac{1-e^{-j 2 \pi L} \frac{m-n}{N}}{j 2 \pi L \frac{m-n}{N}}, \quad \text { if } m \neq n .
\end{array}\right.
$$

This only depends on the distance between the tones $m-n$ and the ratio between the length of the $\mathrm{CP}$ and the number of tones $L / N$. The 1024-tone system can be described by

$$
\left[\begin{array}{c}
y^{(1)} \\
\vdots \\
y^{(16)}
\end{array}\right]=\left[\begin{array}{lll}
X^{(1)} & & \\
& \ddots & \\
& & X^{(16)}
\end{array}\right]\left[\begin{array}{c}
h^{(1)} \\
\vdots \\
h^{(16)}
\end{array}\right]+\left[\begin{array}{c}
n^{(1)} \\
\vdots \\
n^{(16)}
\end{array}\right]
$$

that is, as 16 parallel 64-tone systems

$$
\boldsymbol{y}^{(k)}=\boldsymbol{X}^{(k)} \boldsymbol{h}^{(k)}+\boldsymbol{n}^{(k)}, \quad k=1,2, \cdots, 16 .
$$

We have the same channel correlation in each subsystem as we have in the 64-tone scenario in this paper $(L / N=4 / 64=$ $64 / 1024)$. By estimating the channel attenuations $h^{(k)}$ in each subsystem independently, we neglect the correlation between tones in different subsystems, but obtain the same meansquared error (MSE) performance as in our 64-tone scenario. The advantage is a significant reduction of complexity.

\section{EstIMATOR PeRformance AND DESIGN}

To further simplify this estimator, we propose a generic low-rank frequency-based channel estimator, i.e., an estimator which is designed for fixed nominal values of SNR and channel correlation. Hence, we need to study the choice of the rank, channel correlation, and SNR for this estimator so that it is robust to variations in the channel statistics. As a performance measure, we use uncoded SER for 16-QAM signaling. The SER in this case can be calculated from the MSE with the formulas in [12].

\section{A. SER Performance Under Mismatch}

In practice, the true channel correlation and SNR are not known. To get a general expression for the estimator SER, we derive it under the assumption that the estimator is designed for correlation $R_{h h}$ and SNR, while the true values are $R_{\bar{h} \bar{h}}$ and $\widehat{\mathrm{SNR}}$, respectively, where $\tilde{h}$ denotes a channel with different statistics than $\boldsymbol{h}$. This allows us to analyze this estimator's sensitivity to design errors. Under these assumptions, the MSE (relative to the channel power) of the rank- $p$ estimate (7) becomes (see Appendix D)

$$
\operatorname{mse}(p)=\frac{1}{N} \sum_{k=1}^{p}\left[\mu_{k}\left(1-\delta_{k}\right)^{2}+\frac{\beta}{\widehat{\mathrm{SNR}}} \delta_{k}^{2}\right]+\frac{1}{N} \sum_{k=p+1}^{N} \mu_{k}
$$

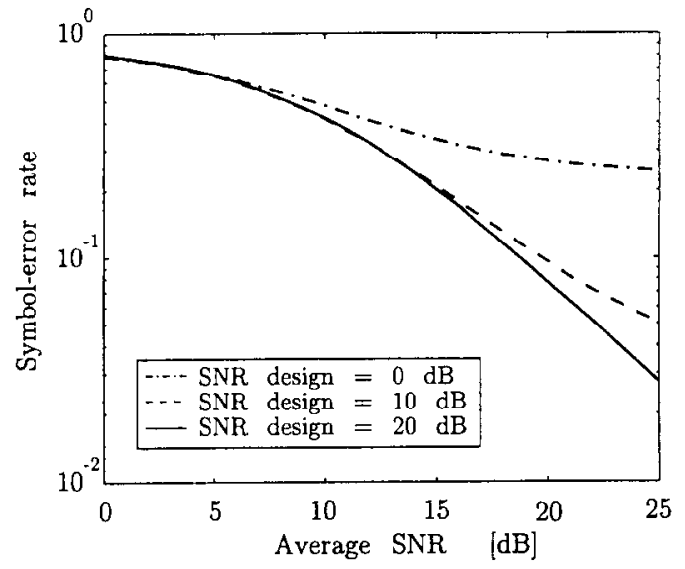

Fig. 5. Effects of SNR design mismatch. The channel estimator is designed for a uniform power-delay profile and evaluated for an exponentially decaying power-delay profile.

where $\delta_{k}$ is defined in (8) and $\mu_{k}$ is the $k$ th diagonal element of $U^{H} R_{\bar{h} \bar{h}} U$, cf. (6). Since

$$
E\left\{\left(U^{H} \tilde{h}\right)\left(U^{H} \tilde{\boldsymbol{h}}\right)^{H}\right\}=U^{H} \boldsymbol{R}_{\tilde{h} \tilde{h}} U
$$

$\mu_{k}$ can be interpreted as the variance of the transformed channel $U^{H} \tilde{\boldsymbol{h}}$ under correlation mismatch. It should be noted that the elements of $U^{H} \tilde{h}$ are no longer uncorrelated. However, due to the fact that the power-delay profile is short compared to the OFDM symbol, the first $p$ elements can be expected to contain most of the power. This property will ensurc only a small performance loss when the estimator is designed for wrong channel statistics.

Fixed FIR estimators have been investigated in [3] and [13], where it is shown that a design for the worst correlation is robust to mismatch. This design rule turns out to hold for lowrank estimators as well. Hence, wc will design the estimator for a uniform power-delay profile [3]. As for mismatch in SNR, a design for a high SNR is preferable. This can intuitively be explained by the fact that a channel estimation error is concealed in noise for low SNR, whereas it tends to dominate for high SNR where the noise is low. Hence, it is important to keep the channel cstimation error low at high SNR, which justifies a design for high SNR. This interpretation is confirmed in Fig. 5, where the SER curves for a design SNR of 0,10 , and $20 \mathrm{~dB}$ are shown.

\section{B. Rank Reduction}

The MSE of the rank- $p$ estimator is mainly determined by the channel power contained in the transform coefficients and can be expressed as (9). The MSE is a monotonically decreasing function of SNR and can be bounded from below by the last term

$$
\underline{\operatorname{mse}}(p)=\frac{1}{N} \sum_{m=p+1}^{N} \mu_{m} \leq \operatorname{mse}(p)
$$

which is the sum of the channel power in the transform coefficients not used in the estimate. This MSE floor $\underline{\operatorname{mse}}(p)$ will cause an irreducible error floor in the SER's. 


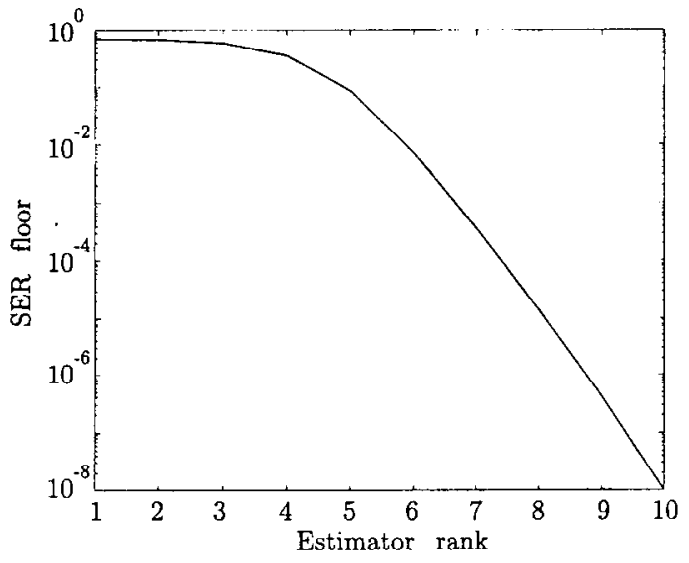

Fig. 6. SER floor as function of estimator rank.

The irreducible error floor is the main limitation on the complexity reduction achieved by optimal rank reduction. The SER floors are shown as a function of the rank in Fig. 6. If the rank is too low, the irreducible error floor will become visible for the SNR of interest. By choosing the appropriate rank on the estimator, we can essentially avoid the impact from the SER floor up to a given SNR. For a full rank estimator $p=N$, no SER floor exists. From Fig. 6, it can be seen that the irreducible error floor decreases rapidly for rank $p>L+1=5$. We are therefore able to obtain a good estimator approximation with a relatively low rank.

\section{GENERIC LOW-RANK ESTIMATOR}

If we want a robust generic channel estimator design for OFDM systems, of the low-rank type, the analysis in the previous section suggests the use of the uniform channel correlation and a relatively high SNR as nominal design parameters. The design of such an estimator only requires knowledge about the length of the $\mathrm{CP}$, the number of tones in the system, and the target range of SNR's for the application. If the receiver cannot afford an estimator that includes tracking of channel correlation and SNR, this channel estimator works reasonably well for fixed SNR and channel correlation.

\section{A. Performance Evaluation}

For the scenario used in this paper, we choose a rank-8 estimator and a design for a uniform power-delay profile and $\mathrm{SNR}=20 \mathrm{~dB}$. The performance of this estimator is presented in Fig. 7, where the SER for the LS estimate (4) and perfect channel knowledge are also shown. The low-rank estimator is $3.5 \mathrm{~dB}$ better than the LS estimator and about $0.8 \mathrm{~dB}$ from perfect channel knowledge.

An alternative to using low-rank cstimators is to use FIR Wiener filters. Their use in OFDM has been investigated in [3]. To make a comparison, we design the FIR Wiener filters for the same channel correlation and SNR as for the lowrank cstimator and use the same complexity. For a rank-8 estimator, we have 16 multiplications per tone and, hence, we can use 16 taps in the FIR Wiener filter. In Fig. 8, the meansquared estimation error is shown for both estimators. As can be seen, the MSE of the low-rank estimator is about 1.5 times

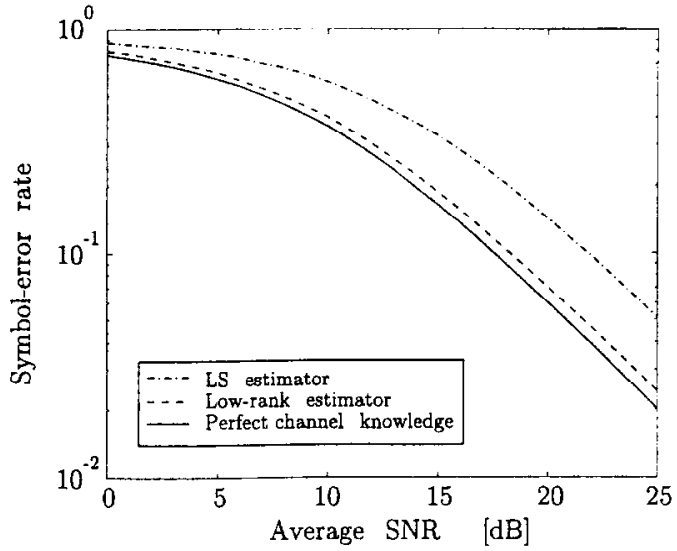

Fig. 7. SER for the low-rank estimator, the LS estimator, and perfect channel knowledge.

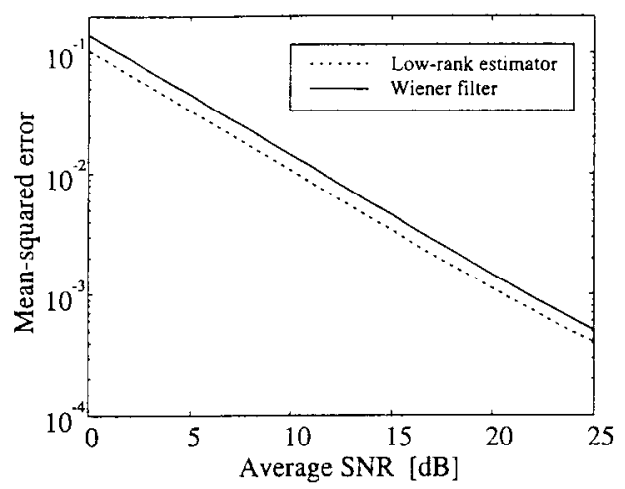

Fig. 8. The MSE of the low-rank estimator (rank $p=8$ ) and the FIR Wiener filter estimator (16 taps). The two estimators have the same computational complexity.

smaller than the MSE of the FIR Wiener filter estimator for all evaluated SNR values.

\section{B. PSAM}

A proposed scheme for cohcrent OFDM is PSAM [3], [14]. In this case known symbols (pilots) are multiplexed into the transmitted data stream and channel estimation is performed by interpolation between these pilots. This interpolation can be performed by FIR Wiener filters [3], but our proposed lowrank estimator is also applicable. In this case our proposed channel estimator is similar to the all-pilots case. The difference is that only those tones on which pilots are transmitted are used for channel estimation, not all tones. The optimal rank reduction is shown in Appendix B and the structure of the estimator is depicted in Fig. 9 (cf. Fig. 4).

The expression for the MSE for this low-rank interpolator will consist of matrix expressions and, so, is more complex than (9). However, the interpolator has similar properties as the estimator (7), and the same design considerations hold. The irreducible error floor is mainly determined by the power of the unused transform coefficients as in (10).

To compare the low-rank estimator with an FIR Wiener filter, we consider a scenario similar to digital video broadcast (DVB) [14]. The system has a bandwidth of $8 \mathrm{MHz}$ and uses 8192 subcarriers with pilot symbols on every twelfth sub- 


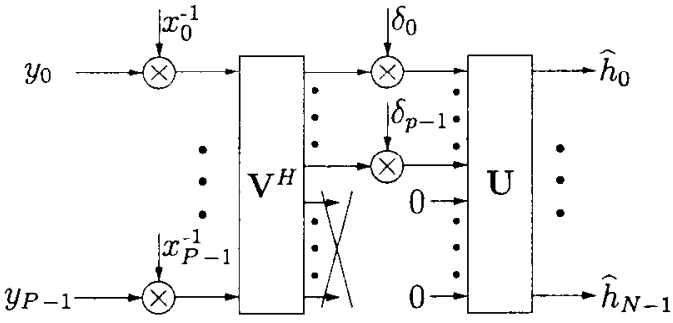

Fig. 9. Low-rank estimator for the PSAM case. For each OFDM symbol, $P$ pilots are used to estimate $N$ tones.

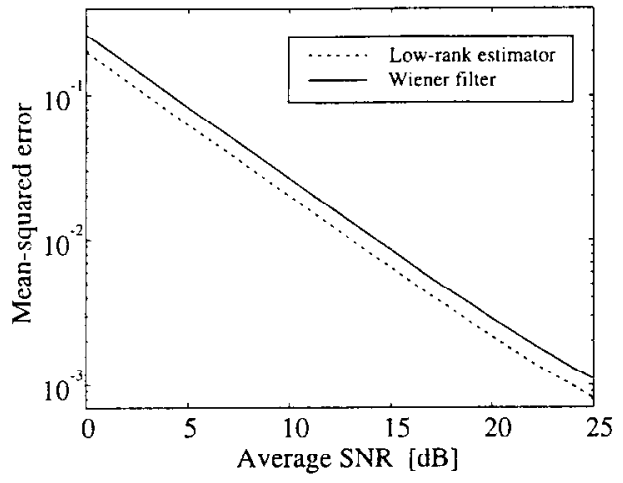

Fig. 10. The MSE of the low-rank estimator and the FIR Wiener filter estimator for the PSAM case. The two estimators have the same computational complexity (eight multiplications per tone)

carrier. Every OFDM symbol is partitioned into subsymbols consisting of 384 subcarriers, each containing $384 / 12=32$ pilots. The power delay profile is exponentially decaying with $\tau_{\text {rms }}=2.5 \mu$ s (20 samples) and a maximum delay spread of $10 \mu \mathrm{s}$ ( $L=80$ samples). The number of coefficients used for the low-rank estimator is seven, resulting in an average complexity of 7.6 multiplications per tone (see Appendix C), and the number of taps for the FIR estimator is eight. As can be seen from the curves in Fig. 10, the MSE of the low-rank estimator is about 1.3 times smaller than the MSE of the FIR estimator for all evaluted SNR values.

\section{The Use of Time Correlation}

The low-rank estimator presented in this paper is based on frequency correlation only, but the time correlation of the channel can also be used. The two-dimensional LMMSE estimator can be simplified using the same technique with rank reduction as described here. However, in [15] it is shown that such an estimator gives an inferior performance for a fixed complexity. Hence, it seems that separating the use of frequency correlation and time correlation is the most efficient way of estimating the channel, as was pointed out in [3].

Other approaches to exploit the time correlation is, e.g., to use a decision-directed scheme [12] or FIR filters [3], [13]. The former can be used in a slow-fading environment, where it offers good performance for a small complexity, whereas the latter is preferred in a fast-fading environment.

\section{CONCLUSIONS}

We have investigated low-complexity low-rank approximations of the LMMSE channel estimator for nonsample- spaced channels. The investigation shows that an estimator error-floor, inherent in the low-rank approximation, is the significant limitation to the achieved complexity reduction. Its performance degradation can be limited by choosing a sufficient rank. We showed that a generic low-rank estimator design, based on the uniform channel correlation and a nominal SNR, can be used in our 64-tone scenario. Compared with the full LMMSE (5) there is only a small loss in performance. up to an SNR of $25 \mathrm{~dB}$, but a reduction in complexity with a factor $N / 2 p=4$. For systems with more subchannels, this gain is even larger. The generic estimator design only requires knowledge of the length of the $\mathrm{CP}$, the number of tones in the system, and the target range of SNR's for the application.

We compared a low-rank estimator to FIR filters across the tones. It was shown that the MSE of the low-rank estimator is about 1.5 times smaller than that of the FIR Wiener filter estimator of equal complexity. Since low-rank estimators are based on subspace projection, it is an advantage if the dimension of the subspace is small. This means that they will improve their performance as the channel length becomes smaller.

The proposed estimator can also be used in PSAM. The principle is the same-the observed channel attenuations are projected onto a (much smaller) subspace where estimation is performed. The final channel estimates are then found by linear combinations of the basis vectors of the subspace. Here the MSE of the low-rank estimator is about 1.3 times smaller than the FIR Wiener filter estimator of equal complexity.

\section{APPENDIX A \\ CHANNEL-CORRELATION MATRICES}

Using the channel model in (2), the attenuation on tonc $k$ becomes

$$
h_{k}=\sum_{i=0}^{M-1} \alpha_{i} e^{-j 2 \pi(k / N) \tau_{i}}
$$

The correlation matrix for the attenuation vector $h$

$$
\boldsymbol{R}_{h h}=E\left\{\boldsymbol{h} \boldsymbol{h}^{I I}\right\} \doteq\left[\bar{r}_{m, n}\right]
$$

can be expressed as $\left(\tau_{k}\right.$ 's independent)

$$
\begin{aligned}
r_{m, n}= & \int \ldots \int \prod_{k=0}^{M-1} f_{\tau_{k}}\left(\tau_{k}\right)\left[\sum_{i=0}^{M-1} \theta\left(\tau_{i}\right) e^{-j 2 \pi \tau_{i}(m-n) / N}\right] \\
& \cdot d \tau_{0} \cdots d \tau_{M-1} \\
= & \sum_{i=0}^{M-1} \int f_{\tau_{i}}\left(\tau_{i}\right) \theta\left(\tau_{i}\right) e^{-j 2 \pi \tau_{i}(m-n) / N} d \tau_{i}
\end{aligned}
$$

where $\theta(\tau)$ is the multipath intensity profile and $f_{\tau_{k}}\left(\tau_{k}\right)$ is the probability density function of $\tau_{k}$.

The probability distributions for the delays are

$$
f_{\tau_{i}}\left(\tau_{i}\right)=\left\{\begin{array}{l}
1 / L, \quad \text { if } \tau_{i} \in[0, L] \\
0, \quad \text { otherwise }
\end{array}, i=0,1, \cdots, M-1\right.
$$


and the power-delay profile is $\theta(\tau)=C e^{-\tau / \tau_{r m s}}$. Substituting in (11) and normalizing $r_{k, k}$ to unity gives us

$$
r_{m, n}=\frac{1-e^{-L\left(\left(1 / \tau_{\mathrm{rms}}\right)+2 \pi j(m-n) / N\right)}}{\tau_{\mathrm{rms}}\left(1-e^{-\left(L / \tau_{\mathrm{rms}}\right)}\right)\left(\frac{1}{\tau_{\mathrm{rms}}}+j 2 \pi \frac{m-n}{N}\right)} .
$$

A uniform power-delay profile can be obtained by letting $\tau_{\text {rms }} \rightarrow \infty$, resulting in

$$
\begin{gathered}
r_{m, n}=\frac{1-e^{-2 \pi j L(m-n) / N}}{2 \pi j L \frac{m-n}{N}} . \\
\text { APPENDIX B } \\
\text { OPTIMAL RANK REDUCTION }
\end{gathered}
$$

\section{OPTIMAL RANK REDUCTION}

The optimal rank reduction is found from the correlation matrices

$$
\begin{aligned}
\boldsymbol{R}_{h \hat{h}_{l s}} & =E\left\{\boldsymbol{h} \hat{\boldsymbol{h}}_{l s}^{H}\right\} \\
\boldsymbol{R}_{\hat{h}_{l s} \hat{h}_{l s}} & =E\left\{\hat{\boldsymbol{h}}_{l s} \hat{h}_{l s}^{H}\right\}
\end{aligned}
$$

and the SVD

$$
R_{h \hat{h}_{l s}} R_{\hat{h}_{l s} \hat{h}_{l s}}^{-1 / 2}=Q_{1} D Q_{2}^{H}
$$

where $Q_{1}$ and $Q_{2}$ are unitary matrices and $D$ is a diagonal matrix with the singular values $d_{1} \geq d_{2} \geq \cdots \geq d_{N}$ on its diagonal. The best rank-p estimator [5] is then

$$
\hat{h}_{p}=Q_{1}\left[\begin{array}{cc}
D_{p} & 0 \\
0 & 0
\end{array}\right] Q_{2}^{H} R_{h_{l s} h_{l s}}^{-1 / 2} \hat{h}_{l s}
$$

where $D_{p}$ is the $p \times p$ upper left corner of $D$, i.e., we exclude all but the $p$ largest singular vectors. For the PSAM case, we have $Q_{1} \neq Q_{2}$, so it is difficult to further reduce (13). However, in the case of all pilots we have $R_{h \hat{h}_{l s}}=R_{h h}$ and $\boldsymbol{R}_{\hat{h}_{l s} \hat{h}_{l s}}=\boldsymbol{R}_{h h}+(\beta / \mathrm{SNR}) \boldsymbol{I}$. We note that they share the same singular vectors, i.e., the ones of $R_{h h}=U \Lambda U^{H}$. Thus, we may express (12) as

$$
\begin{aligned}
R_{h \hat{h}_{l s}} R_{\hat{h}_{l s} \hat{h}_{l s}}^{-1 / 2} & =U \Lambda U\left(U\left(\Lambda+\frac{\beta}{\mathrm{SNR}} I\right) U^{H}\right)^{-1 / 2} \\
& =U \Lambda\left(\Lambda+\frac{\beta}{\mathrm{SNR}} I\right)^{-1 / 2} U^{H} \\
& =Q_{1} D Q_{2}^{H}
\end{aligned}
$$

where

$$
\begin{aligned}
Q_{1} & =Q_{2}=U \\
D & =\Lambda\left(\Lambda+\frac{\beta}{\operatorname{SNR}} I\right)^{-1 / 2} .
\end{aligned}
$$

The rank- $p$ estimator (13) now becomes

$$
\begin{aligned}
\hat{h}_{p}= & U\left[\begin{array}{cc}
D_{p} & 0 \\
0 & 0
\end{array}\right] U^{H} \\
& \cdot\left(U\left(\Lambda+\frac{\beta}{\mathrm{SNR}} I\right) U^{H}\right)^{-1 / 2} \hat{h}_{l s} \\
= & U\left[\begin{array}{cc}
D_{p} & 0 \\
0 & 0
\end{array}\right]\left(\Lambda+\frac{\beta}{\mathrm{SNR}} I\right)^{-1 / 2} U^{H} \hat{h}_{l s} \\
= & U\left[\begin{array}{cc}
\Delta_{p} & 0 \\
0 & 0
\end{array}\right] U^{H} \hat{h}_{l s}
\end{aligned}
$$

where $\Delta_{p}$ is the $p \times p$ upper left corner of

$$
\begin{aligned}
\Delta & =\boldsymbol{\Lambda}\left(\boldsymbol{\Lambda}+\frac{\beta}{\mathrm{SNR}} \boldsymbol{I}\right)^{-1} \\
& =\operatorname{diag}\left(\frac{\lambda_{1}}{\lambda_{1}+\frac{\beta}{\mathrm{SNR}}}, \cdots, \frac{\lambda_{N}}{\lambda_{N}+\frac{\beta}{\mathrm{SNR}}}\right) .
\end{aligned}
$$

\section{APPENDIX C \\ ESTIMATOR COMPLEXITY}

For the all-pilot case, the implementation we have chosen is based on writing (7) as a sum of rank-1 matrices

$$
\hat{\boldsymbol{h}}_{p}=\left(\sum_{k=1}^{p} \delta_{k} \boldsymbol{u}_{k} \boldsymbol{u}_{k}^{H}\right) \hat{\boldsymbol{h}}_{l s}=\sum_{k=1}^{p} \boldsymbol{q}_{k}\left\langle\boldsymbol{u}_{k}, \hat{\boldsymbol{h}}_{l s}\right\rangle
$$

where $\boldsymbol{q}_{k}=\delta_{k} \boldsymbol{u}_{k}$ and $\left\langle\boldsymbol{u}_{k}, \hat{\boldsymbol{h}}_{l s}\right\rangle=\boldsymbol{u}_{k}^{H} \hat{\boldsymbol{h}}_{l s}$ are the Euclidian inner products, requiring $p N$ multiplications. The linear combination of $p$ vectors of length $N$ also requires $p N$ multiplications. The estimation thus requires $2 p N$ multiplications and the total number of multiplications per tone becomes $2 p$.

Similarly, the rank- $p$ PSAM estimator can be formulated as

$$
\hat{\boldsymbol{h}}_{p}=\left(\sum_{k=1}^{p} \boldsymbol{g}_{k} \tilde{\boldsymbol{g}}_{k}^{H}\right) \hat{\boldsymbol{h}}_{l s, \mathrm{pilots}}=\sum_{k=1}^{p} \boldsymbol{g}_{k}\left\langle\tilde{\boldsymbol{g}}_{k}, \hat{\boldsymbol{h}}_{l s, \mathrm{pilots}}\right\rangle
$$

where $\hat{h}_{l s, \text { pilots }}$ contains the LS channel estimates of the $N_{\text {pilots }}$ pilot positions, and $\boldsymbol{g}_{k}$ and $\tilde{\boldsymbol{g}}_{k}$ are vectors of length $N$ and $N_{\text {pilots }}$, respectively. The inner products $\left\langle\tilde{g}_{k}, \hat{h}_{l s, \text { pilots }}\right\rangle$ require $N_{\text {piluts }}$ multiplications each, i.c., a total of $p N_{\text {pilots }}$ multiplications. The linear combination is over $p$ vectors of length $N$, i.e., requires $p N$ multiplications. Sincc $N$ attenuations are simultaneously estimated, the number of multiplications per attenuation becomes

$$
\frac{p N_{\text {pilots }}+p N}{N}=p\left(1+\frac{N_{\text {pilots }}}{N}\right) \text {. }
$$

\section{APPENDIX D \\ ESTIMATOR MSE}

In this appendix we derive the MSE of the rank- $p$ estimator in (7) for the case of all pilots (the data is known to the receiver). We also present the MSE floor, which bounds the achievable MSE from below in low-rank approximations of the LMMSE estimator. To get a general expression for the MSE for the rank- $p$ approximation of the LMMSE estimator, we assume that the estimator has been designed for channel correlation $R_{h h}$ and SNR, but the real channel $\tilde{h}$ has the correlation $R_{\tilde{h} \tilde{h}}$ and the real SNR is SNR. From (1) and (4), we have $\hat{h}_{l s}=\tilde{h}+\tilde{n}$, where the noise term $\tilde{n}=X^{-1} n$ has the autocovariance matrix $R_{i \tilde{n}}=(\beta / \widetilde{\mathrm{SNR}}) I$. The estimation error $e_{p}=\tilde{h}-\hat{h}_{p}$ of the rank-p estimator (7) is

$$
\boldsymbol{e}_{p}=U\left(I-\left[\begin{array}{cc}
\Delta_{p} & 0 \\
0 & 0
\end{array}\right]\right) U^{H} \tilde{h}-U\left[\begin{array}{cc}
\Delta_{p} & 0 \\
0 & 0
\end{array}\right] U^{H_{\tilde{n}}}
$$

and the average MSE is

$$
\operatorname{mse}(p)=\frac{1}{N} \text { Trace } E\left\{\boldsymbol{e}_{p} \boldsymbol{e}_{p}^{H}\right\}
$$


To simplify the expression, we use the facts that:

- $\hat{h}$ and $\tilde{n}$ are uncorrelated, hence the cross terms are cancelled in the expectation;

- Trace $\left(U A U^{H}\right)=$ Trace $\boldsymbol{A}$ if $U$ is a unitary matrix;

- Trace $(\boldsymbol{A}+\boldsymbol{B})=\operatorname{Trace} \boldsymbol{A}+\operatorname{Trace} \boldsymbol{B}$;

- Trace $(D A D)=\Sigma_{k} a_{k, k} \quad d_{k}^{2}$ when $D$ is a diagonal matrix with the elements $d_{k}$ on its diagonal and $A$ (not necessarily a diagonal matrix) has diagonal elements $a_{k, k}$.

Using (15) in (16), the MSE becomes

$$
\begin{aligned}
\operatorname{mse}(p)= & \frac{1}{N} \operatorname{Trace}\left[U\left(I-\left[\begin{array}{cc}
\Delta_{p} & 0 \\
0 & 0
\end{array}\right]\right) U^{I I}\right. \\
& \cdot \boldsymbol{R}_{\tilde{h} \tilde{h}} U\left(I-\left[\begin{array}{cc}
\Delta_{p} & 0 \\
0 & 0
\end{array}\right]\right){ }^{H} U^{H} \\
& \left.+\boldsymbol{U}\left[\begin{array}{cc}
\Delta_{p} & 0 \\
0 & 0
\end{array}\right] U^{H} \boldsymbol{R}_{\tilde{\boldsymbol{n}} \tilde{n}} U\left[\begin{array}{cc}
\Delta_{p} & 0 \\
0 & 0
\end{array}\right]^{H} U^{H}\right] \\
= & \frac{1}{N}\left(\sum_{k=1}^{p} \mu_{k}\left(1-\delta_{k}\right)^{2}+\sum_{k=p+1}^{N} \mu_{k}\right) \\
& +\frac{1}{N} \sum_{k=1}^{p} \frac{\beta}{\widehat{\mathrm{SNR}}} \delta_{k}^{2} \\
= & \frac{1}{N} \sum_{k=1}^{p}\left(\mu_{k}\left(1-\delta_{k}\right)^{2}+\frac{\beta}{\widehat{\mathrm{SNR}}} \delta_{k}^{2}\right) \\
& +\frac{1}{N} \sum_{k=p+1}^{N} \mu_{k}
\end{aligned}
$$

where $\mu_{k}$ is the channel power in the $k$ th transform coefficient, i.e., the $k$ th diagonal element of the matrix $U^{H} \boldsymbol{R}_{\tilde{h} \tilde{h}} U$. The MSE can be lower bounded, $\operatorname{mse}(p) \geq \underline{\operatorname{mse}}(p)$, by what we call the MSE floor

$$
\underline{\operatorname{mse}}(p)=\frac{1}{N} \sum_{k=p+1}^{N} \mu_{k}
$$

If there is no mismatch in SNR or channel correlation, we have $\mu_{k}=$ diagonal elements of $U^{H} R_{h h} U=\lambda_{k}$ and $\widehat{\mathrm{SNR}}=\mathrm{SNR}$, and the MSE becomes

$$
\operatorname{mse}(p)=\frac{1}{N} \sum_{k=1}^{p}\left(\lambda_{k}\left(1-\delta_{k}\right)^{2}+\frac{\beta}{\operatorname{SNR}} \delta_{k}^{2}\right)+\frac{1}{N} \sum_{k=p+1}^{N} \lambda_{k}
$$

\section{REFERENCES}

[1] J. G. Proakis, Digital Communications, 3rd ed. Englewood Cliffs, NJ: Prentice-Hall, 1995.

[2] Radio Broadcasting Systems; Digital Audio Broadcasting (DAB) to Mobile, Portable and Fixed Receivers, ETSI Standard ETS 300401 , Feb. 1995.

[3] P. Höher, "TCM on frequency-selective land-mobile fading channels," in Proc. Tirrenia Int. Workshop Digital Communications, Tirrenia, Italy, Sept. 1991, pp. 317-328.

[4] S. K. Wilson, R. E. Khayata, and J. M. Cioffi, "16-QAM modulation with orthogonal frequency-division multiplexing in a Rayleigh-fading environment," in Proc. IEEE Vehicular Technology Conf., vol. 3, Stockholm, Sweden, June 1994, pp. 1660-1664.

[5] L. L. Scharf, Statistical Signal Processing: Detection, Estimation, and Time Series Analysis. Reading, MA: Addison-Wesley, 1991.
[6] A. Chini, "Multicarrier modulation in frequency selective fading char. nels," Ph.D. dissertation, Carleton Univ., Ottawa, Ont., Canada, 1994.

[7] J. M. Cioffi, private communication, 1994

[8] J. J. van de Beek. O. Edfors, M. Sandell, S. K. Wilson, and P. O. Börjesson, "On channel estimation in OFDM systems," in Proc. IEEE Vehicular Technology Conf., vol. 2, Chicago, IL, July 1995, pp. 815-819.

[9] A. Peled and A. Ruiz, "Frequency domain data transmission using reduced computational complexity algorithms," in Proc. IEEE Int. Conf. Acoustics, Speech, and Signal Processing, Denver, CO, 1980, pp. 964-967.

[10] V. Mignone and A. Morello, "CD3-OFDM: A novel demodulation scheme for fixed and mobile receivers," IEEE Trans. Commun., vol. 44, pp. 1144-1151, Sept. 1996.

[11] H. J. Landau and H. O. Pollak, "Prolate spheriodal wave functions, Fourier analysis and uncertainty -. III: The dimension of the space of essentially time and band-limited signals," Bell Syst. Tech. J., vol. 4l, p. $1295,1962$.

[12] S. K. Wilson, "Digital audio broadcasting in a fading and dispersive channel," Ph.D. dissertation, Stanford Univ., Stanford, CA, Aug. 1994.

[13] J. K. Cavers, "An analysis of pilot-symbol assisted modulation for Rayleigh-fading channels," IEEE Trans. Veh. Technol., vol. 40, pp. 686-693, Nov. 1991.

[14] Digital Broadcasting Systems for Television, Sound and Data Services, ETSI Standard ETS 300744 , Sept. 1996.

[15] M. Sandell and O. Edfors, "A comparative study of pilot-based channil estimators for wireless OFDM," Div. Signal Processing, Luleå Univ. Technology, Luleå, Sweden, Res. Rep. TULEA 1996:19, Sept. 1996.

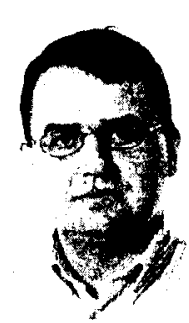

Ove Edfors ( $\left.S^{\prime} 92-A^{\prime} 96\right)$ was born in Ömsköldsvik, Sweden, in 1966. He received the M.Sc. degree in electrical engineering in 1990 and the Ph.D. degree in signal processing in 1996, both from Lule: University of Technology, Luleå, Sweden.

In the spring of 1997 he was with the Division of Signal Processing, Luleå University of Technology, as a Rescarcher, and in July 1997 he joined the staff at the Department of Applied Electronics, Lund University, Lund, Sweden, where he is an Assistant Professor. His research interests include statistical signal processing and low-complexity algorithms with applications in telecommunication.

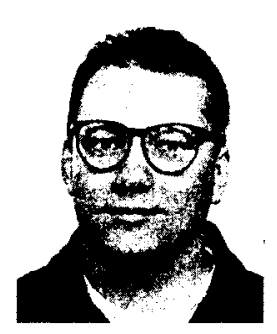

Magnus Sandell ( $S^{\prime}$ 92-A'97) was born in Eksjö, Sweden, in 1965. He received the M.Sc. degree in electrical engineering in 1990 and the Ph.D. degree in signal processing in 1996, both from Luleå University of Technology, Luleå, Sweden.

He spent six months as a Researcher with the Division of Signal Processing, Luleå University of Technology, and then joined Bell Laboratories. Lucent Technologies, Swindon, U.K. His research interests include statistical parameter estimation and algorithms with applications in telecommunication. He primarily works with the physical layer of wireless communication systems.

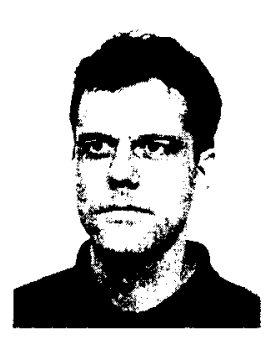

Jan-Jaap van de Beek ( $S^{\prime} 93$ ) was born in Alphen aan den Rijn, The Netherlands, in 1967 . He received the M.S. degree in applied mathematics from the University of Twente, The Netherlands, in 1992. and the Lic. Eng. degree in signal processing frum Luleå University of Technology, Luleå. Sweden. He is currently working toward the Ph.D. degree at the Division of Signal Processing, Luleå University of Technology, Luleå, Sweden.

His research interests include statistical parameter estimation in multicarrier communication systems. 


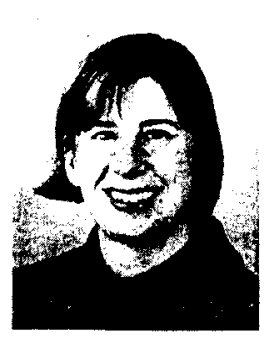

Sarah Kate Wilson (M'87) received the A.B. degree in mathematics from Bryn Mawr College, Bryn Mawr, PA, in 1979, and the M.S. and Ph.D. degrees in electrical engineering from Stanford University, Stanford, CA, in 1987 and 1994.

From 1979 to 1985 she was a Programmer/Analyst with the IIT Research Institute at the Electromagnetic Compatibility Analysis Center. From 1985 to 1986 she was a Research Engineer with SRI International, working with signal processing algorithms for lasar radar systems. From 1987 to 1989 she was a Signal Processing Engineer with Nellcor, Inc., a medical electronics company. She was an Assistant Professor of electrical and computer engineering with Purdue University, West Lafayette, $\mathbb{N}$, from 1994 to 1997. She is currently an Assistant Professor with the Division of Signal Processing, Luleå University of Technology, Luleå, Sweden.

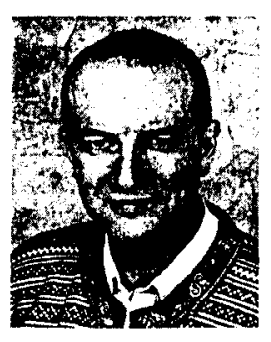

Per Ola Börjesson (S'85-M'80) was born in Karlshamn, Sweden, in 1945. He received the M.Sc. degree in electrical engineering, the Ph.D. degree in telecommunication theory, and the Docent in Telecommunication Theory degree from Lund Institute of Technology, Lund, Sweden, in 1970, 1980, and 1983, respectively.

Since 1988 he has been a Professor of signal processing with Luleå University of Technology, Lulea , Sweden. His primary rescarch interest is in high performance communication systems, in particular, high-data-rate wireless and twisted pair systems. He is presently researching signal processing techniques in communication systems that use OFDM or discrete multitone (DMT) modulation. He emphasizes the interaction between models and real systems from the creation of applicationoriented models based on system knowledge to the implementation and evaluation of algorithms. 